\title{
Estudios citogenéticos y de contenido de ADN en Brasiliopuntia schulzii (Cactaceae)
}

\section{Cytogenetic studies and DNA content in Brasiliopuntia schulzii (Cactaceae)}

\author{
María Laura Las Peñas ${ }^{1 *}$, Federico Santiñaque ${ }^{2}$, Beatriz López-Carro² \& Laura Stiefkens ${ }^{1}$ \\ ${ }^{1}$ Instituto Multidisciplinario de Biología Vegetal (UNC- CONICET), C. C. 495 Córdoba, Argentina. \\ ${ }^{2}$ Instituto de Investigaciones Biológicas Clemente Estable, Avenida Italia 3318. CP. 11600, Montevideo, Uruguay. \\ *laulaspenas@yahoo.com.ar
}

\begin{abstract}
RESUMEN
Se determinaron número cromosómico, cariotipo, patrones de bandeo, localización de genes ribosómicos y contenido de ADN nuclear, en dos poblaciones de Brasiliopuntia schulzii (Cactaceae, subfam. Opuntioideae). La especie resultó diploide $(2 \mathrm{n}=22)$, presentó una fórmula cariotípica de $10 m+1 \mathrm{sm}$. El bandeo cromosómico CMA/DAPI reveló la presencia de un par cromosómico con una banda $\mathrm{CMA}^{+} / \mathrm{DAPI}^{-}$asociada a NORs. Con la técnica de FISH se observó que los loci 185,8-26S fueron consistentes con los bloques $\mathrm{CMA}^{+} / \mathrm{DAPI} / \mathrm{NORs}$. La señal para el gen 5S se localizó en el cuarto par $m$ en una región telomérica. En las dos poblaciones el análisis de contenido de ADN reveló una mezcla de núcleos con tres picos de 2C, 4C y 8C. Esto indicaría un proceso de endopoliploidía en la especie. Los resultados reportados en este trabajo, combinados con las características morfológicas, indicarían que este género posee caracteres basales, concordando con los análisis filogenéticos de la subfamilia.
\end{abstract}

Palabras clave: Brasiliopuntia, cariotipo, contenido de ADN, endopoliploidía, heterocromatina, genes ribosomales.

\begin{abstract}
Chromosome number, karyotype, banding patterns, location of ribosomal genes and nuclear DNA content were determined in two populations of Brasiliopuntia schulzii (Cactaceae, subfam. Opuntioideae). The species resulted diploid $(2 \mathrm{n}=22)$ and presented a karyotype formula of $10 \mathrm{~m}+1 \mathrm{sm}$. The chromosomal banding CMA/DAPI revealed the presence of one chromosome pair with $\mathrm{CMA}^{+} / \mathrm{DAPI}{ }^{-}$band associated to NORs. With FISH technique we observed that 18-5.8-26S loci were consistent with $\mathrm{CMA}^{+} / \mathrm{DAPI} / \mathrm{NORs}$ blocks. The signal for the $5 \mathrm{~S}$ gene is located at the fourth pair $m$ in a telomeric region. In both populations the DNA content analysis revealed a mixture of nuclei with three peaks of $2 \mathrm{C}, 4 \mathrm{C}$ and $8 \mathrm{C}$. This fact suggests a process of endopolyploidy in the species. The results reported in this paper, combined with morphological characteristics, indicate that this genus has basal characters, which coincides with phylogenetic analyses of the subfamily.
\end{abstract}

KEYwords: Brasiliopuntia, DNA content, endopolyploidy, heterochromatin, karyotype, ribosomal genes.

\section{INTRODUCCIÓN}

Brasiliopuntia A. Berger es un género que incluye dos especies (Kiesling et al. 2008). El género reúne varios caracteres basales dentro de las Opuntioideae como la forma arbórea, tronco cilíndrico sin articulaciones, los frutos grandes muy carnosos con pocas semillas (Pin 1998). La diferencia entre las dos especies está dada principalmente por el fruto. Brasiliopuntia schulzii (A. Cast. \& Lelong) Backeb. se caracteriza por poseer frutos piriformes, rojos, con aréolas pequeñas con muchos gloquidios, caducos a la maduración, al observar sólo ejemplares de herbario no se ven gloquidios, o se observan pocos. B. brasiliensis (Willd.) A. Berger posee frutos globosos, amarillos, con aréolas grandes con gloquidios muy sobresalientes que se mantienen a la madurez en forma de mechones (Pin 1998). Brasiliopuntia schulzii se distribuye al este y sur de Paraguay y noreste de Argentina, en cambio B. brasiliensis se distribuye al este de Brasil, Bolivia y Perú y Norte de Argentina (Pin 1998).

Los análisis de filogenia molecular apoyan la permanencia de Brasiliopuntia dentro de la tribu Opuntieae 
(platiopuntias) incluyendo a los géneros Consolea Lem., Miqueliopuntia Frič ex F.Ritter, Nopalea Salm-Dyck y Opuntia Mill. sensu stricto (Wallace \& Dickie 2002, Griffith \& Porter 2009, Hernández-Hernández et al. 2011).

La mayoría de los estudios citogenéticos en Cactaceae indican que su número básico es $\mathrm{x}=11$ (cf. Pinkava et al. 1985, 1998, Powell \& Weedin 2001, Pinkava 2002, Goldblatt \& Johnson 2013). A la fecha se conocen los números cromosómicos y cariotipos de varias especies de la subfamilia Opuntioideae de América del Norte (Bandyopadhyay \& Sharma 2000, Palomino \& Heras 2001).

Para Brasiliopuntia se ha reportado un recuento cromosómico en $B$. brasiliensis $2 \mathrm{n}=22$ (Pereira de Castro 2008), no hay datos disponibles con respecto a sus características cariotípicas.

La técnica de bandeo cromosómico fluorescente CMA/ DAPI se utiliza para examinar la presencia, tipo y distribución de heterocromatina (HC) utilizando fluorocromos base específicos, como cromomicina $\mathrm{A}_{3}$ (CMA) y 4'-6-diamidino2-fenilindol (DAPI). Las bandas obtenidas generalmente se encuentran asociadas a constricciones secundarias en angiospermas (Souza \& Benko-Iseppon, 2004, Blanco et al. 2012, Chiarini et al. 2014). Esta técnica fue utilizada en especies sudamericanas de Cactaceae (Las Peñas et al. 2008, 2009, 2011, 2014).

El empleo de la técnica de hibridación in situ fluorescente (FISH) de diferentes secuencias de ADN permite marcar uno o varios genes en los cromosomas del complemento y así establecer nuevos tipos de marcadores cromosómicos para un análisis detallado del cariotipo (Maluszynska \& Heslop-Harrison 1993). Esta técnica ha sido utilizada en varios géneros de angiospermas (Moscone et al. 2007, Blanco et al. 2012, Moreno et al. 2012). En Cactaceae se ha aplicado en 12 especies (Las Peñas et al. 2009, 2011, 2014).

Desde el punto de vista citogenético y de evolución cromosómica, la cuantificación del contenido de ADN en especies relacionadas constituye un elemento adicional para analizar los cambios ocurridos durante o con posterioridad al proceso de especiación. Así, la determinación del valor $\mathrm{C}$ (cantidad de ADN de un genoma haploide no replicado de un individuo) ha permitido detectar variaciones entre especies con cariotipos aparentemente similares (Ohri 1998, Bottini 2000, Las Peñas et al. 2014).

El método más usado actualmente es la citometría de flujo (CF; Costich et al. 1991, Doležel 1991). En Opuntioideae existen algunos estudios previos de medición de ADN nuclear en especies de los géneros Consolea y Opuntia (Palomino \& Heras 2001, Zonneveld et al. 2005, NegronOrtiz 2007, Segura et al. 2007). El valor Cx (contenido de ADN por genoma básico) en las especies de la subfamilia varía desde 0,54 a 3,46 pg.

Sobre estos antecedentes, el objetivo de este trabajo fue estudiar los cromosomas mitóticos con técnica clásica de bandeo cromosómico fluorescente y FISH, y cuantificar el tamaño del genoma en dos poblaciones argentinas de B. schulzii a fin de aportar datos para entender su sistemática y relaciones evolutivas con los demás géneros de Opuntioideae.

\section{MATERIALES Y MÉTODOS}

Se estudiaron dos poblaciones de Brasiliopuntia schulzii de Argentina provincia de Corrientes: Población 1, Ciudad de Corrientes, Perichón Las Peñas 193; Población 2, Ciudad de Corrientes, Las Peñas et al. 523. Las plantas fueron cultivadas en la Facultad de Ciencias Exactas, Físicas y Naturales (IMBIV-UNC), los ejemplares se encuentran depositados en el Museo Botánico de Córdoba (CORD).

Los estudios mitóticos se realizaron en ápices radicales pretratados en 8-hidroxiquinoleína $0,002 \mathrm{M}$ durante $24 \mathrm{~h}$ a $4^{\circ} \mathrm{C}$, que posteriormente se fijaron en solución Farmer (3:1; alcohol etílico: ácido acético glacial) y se conservaron a -20 ${ }^{\circ} \mathrm{C}$. Posteriormente a la fijación, las raíces se hidrolizaron con ácido clorhídrico $5 \mathrm{~N}$ durante $30 \mathrm{~min}$ a temperatura ambiente, luego lavadas con agua destilada durante $5 \mathrm{~min}$ y por último coloreadas con reactivo de Schiff por $2 \mathrm{~h}$ a temperatura ambiente en oscuridad (Jong 1997). Los meristemas fueron aplastados en ácido acético $45 \%$ y luego los preparados se hicieron permanentes removiendo el cubreobjetos por congelamiento con nitrógeno líquido $\left(\mathrm{N}_{2}\right)$ y montando con Euparal. Los cariogramas fueron construidos siguiendo la nomenclatura de Levan et al. (1964). La asimetría del cariotipo fue estimada usando los índices de asimetría $\mathrm{A}_{1} \mathrm{y}$ $\mathrm{A}_{2}$ propuesto por Romero Zarco (1986).

Para el bandeo cromosómico fluorescente CMA/DAPI y FISH las preparaciones cromosómicas se llevaron a cabo mediante digestión enzimática de las raíces con Pectinex ${ }^{\circledR}$ a $37^{\circ} \mathrm{C}$ durante 30 minutos y luego fueron aplastadas con ácido acético $45 \%$, posteriormente los cubreobjetos se retiraron por congelamiento con nitrógeno líquido.

La técnica utilizada para el bandeo fue doble tinción fluorescente cromomicina $\mathrm{A}_{3}$ (CMA)/ 4'-6-diamidino2-fenilindol (DAPI) según el protocolo descripto por Schweizer \& Ambros (1994) con pequeñas modificaciones: los preparados fueron teñidos con CMA por $90 \mathrm{~min}$, lavados con agua destilada y posteriormente teñidos con DAPI por $30 \mathrm{~min}$, lavados con agua destilada y finalmente montados con buffer MacIIvaine: glicerol (1:1). Se conservaron en oscuridad durante 3 a 5 días a $4^{\circ} \mathrm{C}$ para luego ser observados con microscopio de fluorescencia.

La técnica de FISH se realizó siguiendo el protocolo descrito por Schwarzacher \& Heslop-Harrison (2000). Como sondas se utilizaron regiones de ADN ribosómico 185,8-26S (pTa71, Gerlach \& Bedbrook 1979) y 5S obtenida a partir del genoma de Pereskia aculeata por amplificación por PCR utilizando partidores específicos. Los fragmentos de ADN utilizados como sonda fueron marcados con biotina 
(Bionick, Invitrogen) y digoxigenina (DIG Nick translation mix, Roche) mediante la técnica de nicktranslation. Las señales de hibridación fueron detectadas utilizando avidina-FITC (Sigma) y anti-DIG-rodamina (Roche), los cromosomas se colorearon con DAPI.

Las fotomicrografías fueron tomadas en microscopio Zeiss Axiophot y cámara digital Leica DFC300FX. Para la adquisición de fotos de coloración convencional se utilizó contraste de fases, en tanto que para las de bandeo cromosómico y FISH se utilizó epifluorescencia con los filtros correspondientes para cada fluorocromo. Las bandas heterocromáticas obtenidas con bandeo de fluorescencia fueron medidas en 2-5 placas metafásicas correspondientes a 2-5 individuos de cada población. Se calculó el porcentaje de heterocromatina asociada a regiones organizadoras nucleolares ( $\%$ HC-NORs) en relación al largo total del genoma, utilizando el programa Image J (NIH ImageJ; National Institutes of Health, Bethesda, MD, desarrollado por: National Institutes of Health y disponible en el sitio http://rsb.info.nih.gov/ij/). Estos datos se adicionaron a los idiogramas construidos en base a las mediciones cromosómicas de metafases teñidas con Feulgen. Por otro lado, las señales obtenidas mediante la técnica de FISH también fueron localizadas en estos idiogramas.

Para calcular el contenido de ADN nuclear se siguió la metodología de Doležel et al. (2007) con pequeñas modificaciones. En una cápsula de Petri conteniendo $0,5 \mathrm{ml}$ de la solución de Otto I (ácido cítrico $0,1 \mathrm{M}$ y $0,5 \%$ de Tween 20) y $0,2 \mathrm{ml}$ de $1 \%$ de PVP, se colocaron $100 \mathrm{mg}$ de tejido caulinar de B. schulzii y $25 \mathrm{mg}$ de tejido foliar de Zea mays L. CE - 777 (2C = 5,43 pg), utilizado como estándar interno. El material se trozó finamente con hoja de afeitar y se filtró a través de una malla de nylon de $45 \mu \mathrm{m}$ para separar la suspensión de núcleos del material grueso. La suspensión de núcleos se incubó por 10 a 60 min a temperatura ambiente; luego se adicionó $0,5 \mathrm{ml}$ de tampón de Otto II (0,4 M de $\left.\mathrm{Na}_{2} \mathrm{HPO}_{4} 12 \mathrm{H}_{2} \mathrm{O}\right)$ y se realizó un nuevo filtrado. Se agregaron $125 \mu \mathrm{l}$ de ARNasa e igual cantidad de yoduro de propidio, para su análisis en el CF (BectonDickinson, San José, EEUU), sintonizado para emitir luz a $488 \mathrm{~nm}$.

Se llevaron a cabo tres estimaciones de ADN para cada población (5.000 núcleos por análisis), en tres días diferentes. El contenido de ADN nuclear 2C en pg, se calculó como pico promedio de la muestra/ pico promedio estándar.

Las diferencias en el contenido de ADN entre las dos poblaciones fueron verificadas a través de un análisis de varianza (ANOVA) y las comparaciones entre las medias fueron realizadas utilizando el test de Tukey.

TABla I. Brasiliopuntia schulzii, resultados cariotípicos. s, brazo corto; 1, brazo largo; c, largo total; r, índice braquial (r=1/s); i, índice centromérico $(\mathrm{i}=100 \mathrm{~s} / \mathrm{c})$. Las longitudes medias cromosómicas están expresadas en $\mu \mathrm{m}$ ( \pm desvío estándar). Abreviaturas según Levan et al. (1964).

TABLE I. Brasiliopuntia schulzii, karyotypic result. s, short arms; 1, long arms; c, total length; $r$, arm ratios ( $r=1 / s)$; i, centromeric indices (i= $100 \mathrm{~s} / \mathrm{c})$. Measurements in $\mu \mathrm{m}$ ( \pm standard deviation). Abbreviations after Levan et al. (1964).

\begin{tabular}{lccccc}
\hline PAR & $\mathrm{s}$ & 1 & $\mathrm{c}$ & $\mathrm{r}$ & $\mathrm{i}$ \\
\hline 1 & $1,25 \pm 0,01$ & $1,41 \pm 0,03$ & $2,66 \pm 0,62$ & 1,12 & 47,09 \\
2 & $1,14 \pm 0.02$ & $1,22 \pm 0,04$ & $2,36 \pm 0,69$ & 1,06 & 48,41 \\
3 & $1,07 \pm 0.07$ & $1,21 \pm 0,07$ & $2,28 \pm 0,79$ & 1,12 & 47,04 \\
4 & $1,04 \pm 0,01$ & $1,19 \pm 0,01$ & $2,23 \pm 0,76$ & 1,13 & 46,75 \\
5 & $0,91 \pm 0,03$ & $1,19 \pm 0,04$ & $2,10 \pm 0,78$ & 1,31 & 43,23 \\
6 & $0,92 \pm 0,06$ & $1,12 \pm 0,07$ & $2,04 \pm 0,79$ & 1,21 & 45,09 \\
7 & $0,89 \pm 0,07$ & $1,07 \pm 0,07$ & $1,96 \pm 0,80$ & 1,20 & 45,40 \\
8 & $0,91 \pm 0,07$ & $1,03 \pm 0,08$ & $1,94 \pm 0,85$ & 1,13 & 46,78 \\
9 & $0,90 \pm 0,08$ & $0,97 \pm 0,07$ & $1,87 \pm 0,83$ & 1,07 & 48,26 \\
10 & $0,84 \pm 0,08$ & $0,95 \pm 0,07$ & $1,79 \pm 0,91$ & 1,13 & 46,79 \\
11 & $0,75 \pm 0,02$ & $1,29 \pm 0,03$ & $2,05 \pm 0,88$ & 1,71 & 36,83 \\
\hline
\end{tabular}



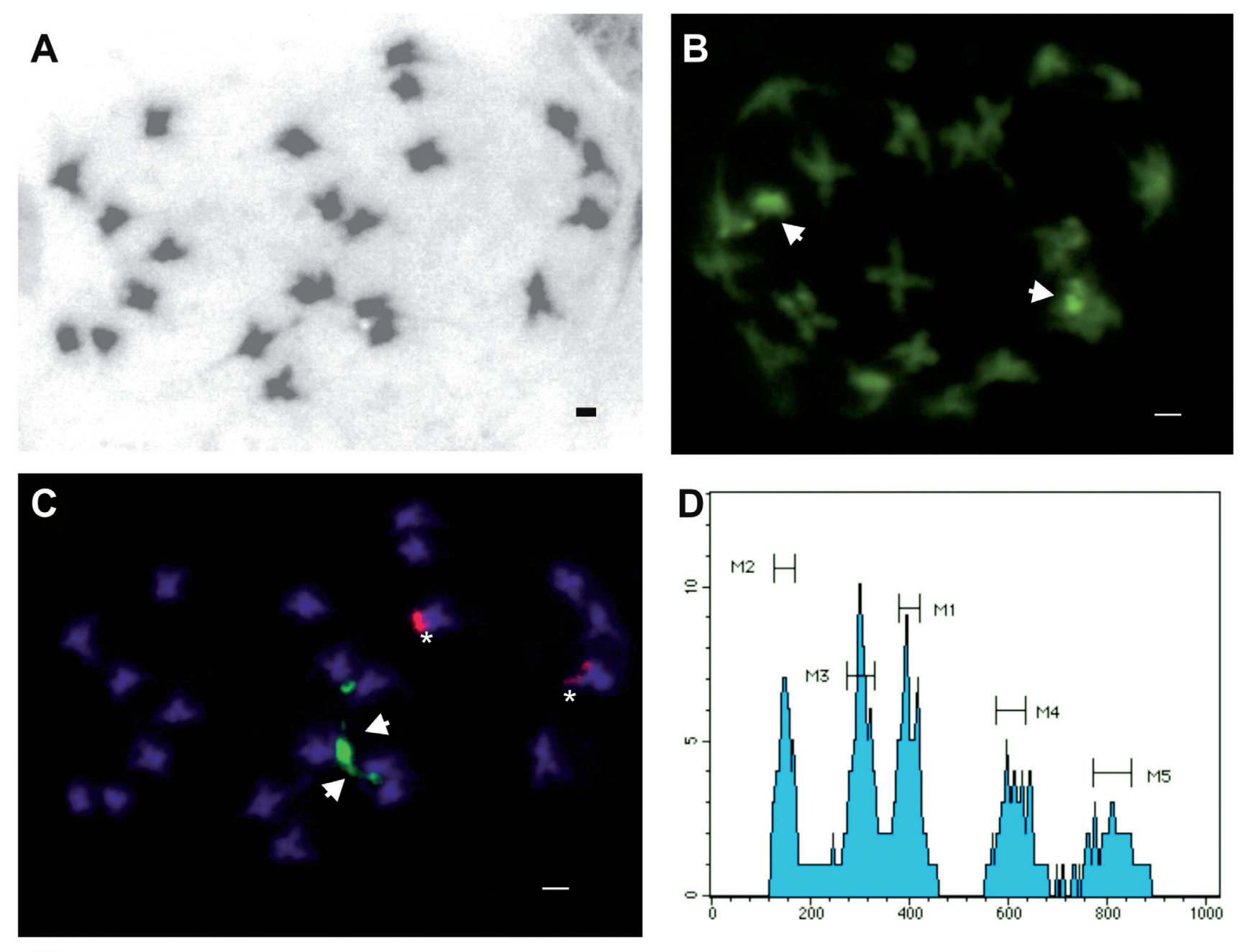

E

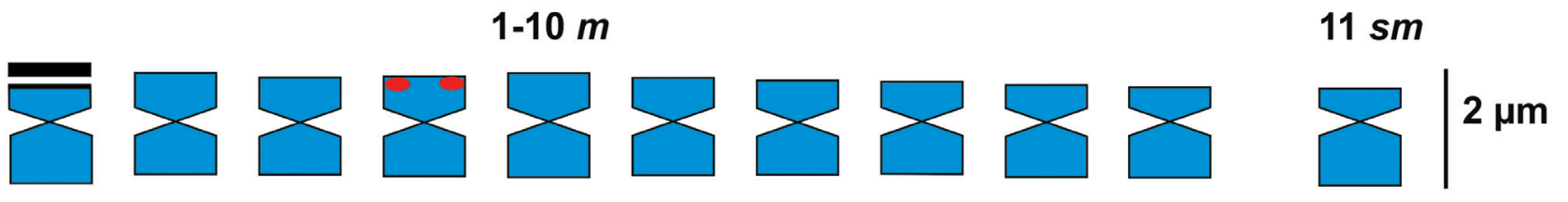

Figura 1. Brasiliopuntia schulzii. A. Metafase mitótica realizada con tinción de Feulgen $(2 \mathrm{n}=22)$. B. Bandeo cromosómico CMA. Las flechas indican regiones $\mathrm{CMA}^{+}$/DAPI ${ }^{-}$. C. FISH usando sondas de ADNr 18S-5,8-26S y 5S, los asteriscos muestran regiones portadoras del locus 5S, las flechas indican sitios 18-5,8-26S en regiones terminales del cromosoma portador y la cabeza de flecha los satélites. D. Histograma del contenido de ADN nuclear en picogramos por citometría de flujo, los picos M1 y M5 representan los núcleos en G1 y G2 de Zea mays (planta de referencia); los picos M2, M3 y M4 representan los valores de 2C, 4C y 8C, de la especie estudiada. E. Idiograma. Color negro indica sitios 18-5,8-26S y regiones $\mathrm{CMA}^{+} / \mathrm{DAPI}$, color rojo muestra regiones portadoras de $5 \mathrm{~S}$. Barra $=2 \mu \mathrm{m}$.

Figure 1. Brasiliopuntia schulzii. A. Somatic metaphase with Feulgen staining $(2 n=22)$. B. Fluorochrome banding with CMA fluorescence. C. FISH using $18 \mathrm{~S}-5.8-26 \mathrm{~S}$ and $5 \mathrm{~S}$ rDNA probes. Asterisks show the locus 5S, arrows indicate the sites 18-5.8-26S carrier terminal chromosome and arrowhead satellites D. Histogram of nuclear DNA content by flow cytometry in picograms, peaks M1 and M5 represent G1 and G2 nuclei of Zea mays (reference plants); M2, M3 and M4 peaks represent the values of 2C, 4C and 8C of the species tested. E. Idiogram. Black color indicates regions 18-5.8-26S and $\mathrm{CMA}^{+} / \mathrm{DAPI}^{-}$, red color shows regions which 5S sites. Bar $=2 \mu \mathrm{m}$. 


\section{RESULTADOS}

Brasiliopuntia schulzii presentó un número cromosómico de $2 n=22$ en las poblaciones analizadas (Fig. 1A). Los cromosomas son pequeños con un largo promedio de $2,12 \mu \mathrm{m}$ y la media del genoma haploide de $23,32 \mu \mathrm{m}$. La especie presentó una fórmula cariotípica de $10 \mathrm{~m}+1 \mathrm{sm}$. En la Tabla I se incluyen las características cariotípicas para cada par cromosómico que fueron usadas para generar el idiograma (Fig. 1E). Con la técnica clásica no fue posible observar satélites. El cariotipo fue simétrico con pequeñas diferencias entre los pares cromosómicos, presentando un índice de asimetría intracromosómico $\left(\mathrm{A}_{1}\right)$ de 0,15 y un intercromosómico $\left(\mathrm{A}_{2}\right)$ de 0,11 .

Con la técnica de bandeo cromosómico CMA/DAPI se observó la presencia de un par cromosómico con una banda $\mathrm{CMA}^{+} / \mathrm{DAPI}$ asociada a NORs (primer par $m$ ) y en parte del brazo que lo porta (Fig. 1B). El porcentaje de heterocromatina $\mathrm{CMA}^{+} / \mathrm{DAPI}^{-}$asociado a NORs fue de 3,18 $\%$ del total del genoma haploide. Por último, se mapearon los genes ribosómicos (FISH), la señal de hibridación para el gen 18-5,8-26S fue localizada en el primer par $m$, observada en el brazo en la región terminal y en la constricción secundaria. Las constricciones secundarias del par cromosómico se encuentran adheridas entre ellas (Fig. $1 \mathrm{C})$. La señal para el gen $5 \mathrm{~S}$ se localizó en el cuarto par $m$ en una región telomérica (Fig. 1C). El tamaño, número e intensidad de las señales de ambos loci $\mathrm{ADNr}$ fueron similares entre los homólogos (Fig. 1B, C, E).

En la Tabla II se resumen los datos de mediciones de contenido de ADN nuclear total en picogramos obtenidos para las poblaciones de B. schulzii (Fig. 1D). En las dos poblaciones el análisis citométrico reveló una mezcla de núcleos con tres picos de $2 \mathrm{C}=2,11$ y $2,08,4 \mathrm{C}=4,22$ y $4,28,8 \mathrm{C}=8,66$ y 8,58 . Esto indicaría un proceso de endopoliploidía en la especie. Estos resultados constituyen el primer reporte de contenido de ADN nuclear para el género. Al realizar un análisis ANOVA se observó que no hubo diferencias significativas en el contenido de ADN (2C, $4 \mathrm{C}, 8 \mathrm{C}$ ) entre las poblaciones estudiadas.

TABLA II. Brasiliopuntia schulzii. Valores promedio estimados de ADN nuclear para las dos poblaciones, pg \pm desvío estándar.

TABLE II. Brasiliopuntia schulzii. Average estimated values of nuclear DNA for the two populations, $p g \pm$ standard deviation.

\begin{tabular}{lccc}
\hline Población & $2 \mathrm{C}$ & $4 \mathrm{C}$ & $8 \mathrm{C}$ \\
\hline 1 & $2,11 \pm 0,03$ & $4,22 \pm 0,09$ & $8,66 \pm 0,10$ \\
2 & $2,08 \pm 0,03$ & $4,28 \pm 0,08$ & $8,58 \pm 0,07$ \\
\hline
\end{tabular}

\section{DISCUSIÓN Y CONCLUSIONES}

Cactaceae se caracteriza por poseer un número básico de $\mathrm{x}=$ 11 (e.g., Bandyopadhyay \& Sharma 2000, Pinkava 2002, Das \& Mohanty 2006, Las Peñas et al. 2009, 2011). El 65\% de las especies de Opuntioideae estudiadas hasta el momento son poliploides (Pinkava 2002), sin embargo las dos especies de Brasiliopuntia son diploides con $2 \mathrm{n}=22$ (B. brasilensis Pereira de Castro 2008 y B. schulzii en este trabajo).

En B. schulzii los cromosomas resultaron pequeños, el cariotipo fue simétrico, encontrándose que la mayoría de los pares cromosómicos resultaron $m$ y entre ellos no se observaron grandes diferencias en el tamaño $\left(A_{1}\right.$ y $\left.A_{2}\right)$. Todo esto lo hace muy semejante a los cariotipos de las otras especies de Opuntioideae ya estudiadas (Bandyopadhyay \& Sharma 2000, Palomino \& Heras 2001) y también a especies de las otras subfamilias de Cactaceae (Cota \& Philbrick 1994, Bandyopadhyay \& Sharma 2000, Las Peñas et al. $2008,2009,2014)$. Teniendo en cuenta estos datos se podría afirmar que la especiación en la familia no está acompañada de grandes cambios estructurales en el cariotipo y esto le permite a las especies hibridar entre ellas en los ambientes naturales (Pinkava 2002).
En Brasiliopuntia schulzii la diferencia de tamaños cromosómicos entre los pares es pequeña; de acuerdo a las mediciones el primer par $m$ es el portador de la banda $\mathrm{CMA}+/$ DAPI- asociada a NOR. Según Guerra (2000) la mayoría de las especies tienen un par de bandas CMA+/ DAPI- que se encuentran asociadas a NOR, ya que todos ellos tienen al menos un par de NORs. Este tipo de heterocromatina es característico en la familia (Las Peñas et al. 2008, 2011, 2014) y en angiospermas (Guerra 2000, Blanco et al. 2012, Moreno et al. 2012).

La localización de los sitios de 18-5,8-26S en B. schulzii se correlacionó siempre con las bandas de heterocromatina $\mathrm{CMA}^{+} / \mathrm{DAPI}^{-}$asociadas a NORs. Tal ubicación está altamente conservado en la familia Cactaceae (Las Peñas et al. 2009, 2014) y también ha sido reportada en Asteraceae y Solanaceae (Blanco et al. 2012, Moreno et al. 2012). Además, se reportó la localización de $\mathrm{ADNr} 5 \mathrm{~S}$ en posición telomérica.

Los valores obtenidos de contenido de ADN nuclear mostraron una mezcla de núcleos con tres picos de $2 \mathrm{C}$, $4 \mathrm{C}$ y $8 \mathrm{C}$ para la especie, determinándose que ésta presenta endopoliploidía. Estos resultados concuerdan con los datos previos para la subfamilia Opuntioideae (Hunt et al. 
2006), solamente en 15 de 200 especies se ha analizado el tamaño del genoma, con un promedio de Cx de ADN 3,14 pg (Bennett \& Leitch 2010), y existen varios registros de especies con procesos de endopoliploidía (Negron-Ortiz 2007, Segura et al. 2007). Este es un proceso en el que las células se someten a ciclos repetidos de síntesis de ADN, multiplicándose el número de cromosomas sin división celular, resultando así una multiplicación exponencial del contenido de ADN nuclear. Este proceso puede variar entre los individuos de una misma especie en respuesta a diferentes condiciones ambientales (Barow 2006, Leitch \& Leitch 2013). Algunos autores han indicado que la endopoliploidía es beneficiosa para los cactus por poseer un tamaño pequeño del genoma (Palomino et al. 1999, Del Angel et al. 2006). Este mecanismo se considera ecológicamente importante como una adaptación a altas temperaturas y escasez de agua (Nagl 1978, Negron-Ortiz 2007, Leitch \& Leitch 2013).

El rango de ADN Cx en las Opuntioideae es 0,55-3, 46 pg (media: 0,93 $\pm 0,61 \mathrm{pg}$ ) (Negron-Ortiz 2007, Segura et al. 2007, Leitch \& Leitch 2013). El contenido Cx encontrado en B. schulzii $(1,04 \mathrm{pg})$ es comparativamente más alto que la media registrada hasta el momento en la subfamilia. Es importante remarcar que los datos previos pertenecen a especies de Norteamérica (Segura et al. 2007, Leitch \& Leitch 2013), por lo que sería importante seguir estudiando otras especies de la subfamilia Opuntioideae de Sudamérica.

Según el análisis estadístico realizado (ANOVA) se observó que no hay diferencias significativas entre las dos poblaciones en el contenido de ADN 2C, 4C y 8C.

Según los análisis filogenéticos de la subfamilia Opuntioideae (Griffith \& Porter 2009, Majure et al. 2012), Brasiliopuntia tiene una posición basal. Teniendo en cuenta los datos aquí obtenidos, se podría inferir que el número cromosómico diploide, el cariotipo simétrico y el bajo porcentaje de heterocromatina corresponderían a características basales que también se mantendrían en algunos grupos derivados.

\section{AGRADECIMIENTOS}

Al Dr. Roberto Kiesling por el envío de material (población 1, Las Peñas 193). Los autores desean agradecer a la asistencia del Consejo Nacional de Investigaciones Científicas y Técnicas (CONICET) y la Universidad Nacional de Córdoba, ambas instalaciones de apoyo utilizados en esta investigación. CONICET, FONCyT y SECYT-UNC brindaron soporte financiero para realizar el presente trabajo.

\section{BIBLIOGRAFÍA}

Bandyopadhyay, B. \& A. Sharma. 2000. The use of multivariate analysis of karyotypes to determine relationships between species of Opuntia. Caryologia 53: 121-126.

BArow, M. 2006. Endopolyploidy in seed plants. BioEssays 28: 271-281.

Bennett, M.D. \& I.J. Leitch. 2010. Plant DNA C-values Database (release 5.0, December 2010). Available on-line: http:// data.kew.org/cvalues/ (last access 15 Aug. 2011).

Blanco, S., M.L. Las Peñas, G. Bernardello \& L. Stiefkens. 2012. Mapeo de genes ribosómicos y heterocromatina en seis especies de Lycium de Sudamérica (Solanaceae). Boletín de la Sociedad Argentina de Botánica 47: 389-399.

Bottini, M.C.J. 2000. Estudios multidisciplinarios en las especies patagónicas argentinas del género Berberis L. (Berberidaceae). Tesis doctoral. Universidad de Buenos Aires, Buenos Aires, Argentina. 223 pp.

Chiarini, F., F. Santiñaque, J. Urdampilleta \& M.L. Las Peñas. 2014. Genome size and karyotype diversity in Solanum sect. Acanthophora (Solanaceae). Plant Systematic and Evolution 300: 113-125.

Costich, D.E., T.R. Meagher \& E.J. Yurkow. 1991. A rapid means of sex identification in Silene latifolia by use of flow cytometry. Plant Molecular Biology Reporter 9: 359-370.

Cota, J.H. \& C.T. Philbrick. 1994. Chromosome number variation and polyploidy in the genus Echinocerus (Cactaceae). American Journal of Botany 81: 1054-1062.

Das, A.B. \& S. Mohanty. 2006. Karyotype analysis and in situ nuclear DNA content in seven species of Echinopsis Zucc. of the family Cactaceae. Cytologia 71: 75-79.

Del Angel, C., G. Palomino, A. Garcia \& I. Mendez. 2006. Nuclear genome size and karyotype analysis in Mammillaria species (Cactaceae). Caryologia 59: 177-186.

DoležEL, J. 1991. Flow cytometric analysis of nuclear DNA content in higher plants. Phytochemical Analysis 2: 143-154.

Doležel, J., J. Greilhuber \& J. Suda. 2007. Estimation of nuclear DNA content in plants using flow cytometry. Nature Protocols 2: 2233-2244.

Gerlach, W.L. \& J.L. Bedbrook. 1979. Cloning and characterization of ribosomal RNA genes from wheat and barley. Nucleic Acids Research 7: 1869-1885.

Goldblatt, P. \& D.E. Johnson. 2013. Index to plant chromosome numbers. 1979 onwards. [Online.] Available from http:// www.tropicos.org/Project/IPCN.

Griffith, M.P. \& J.M. Porter. 2009. Phylogeny of Opuntioideae (Cactaceae). International Journal of Plant Sciences 170: 107-116.

Guerra, M. 2000. Patterns of heterochromatin distribution in plant chromosome. Genetics and Molecular Biology 23: 10291041 .

Hernández-Hernández, T., H.M. Hernández, J.A. De-Nova, R. Puente, L.E. Eguiarte \& S. Magallon. 2011. Phylogenetic relationships and evolution of growth form in Cactaceae (Caryophyllales, Eudicotyledoneae). American Journal of Botany 98: 44-61.

Hunt, D., N. Taylor \& G. Charles. 2006. The New Cactus Lexicon. Milborne Port. DH Books, Milborne Port. 526 pp. 
JoNG, J. 1997. Laboratory manual of plant cytologycal techniques. Royal Botanical Garden, Edinburgh. 97 pp.

Kiesling, R., L. Larrocca, J. Faúndez, D. Metzing \& S. Albesiano. 2008. Cactaceae. En: F.O. Zuloaga, O. Morrone \& M.J. Belgrano (eds.), Catálogo de las Plantas Vasculares del Cono Sur. Monographs in Systematic Botany from the Missouri Botanical Garden 107, pp. 1715-1830.

Las Peñas, M.L., G. Bernardello \& R. Kiesling. 2008. Karyotypes and fluorescent chromosome banding in Pyrrhocactus (Cactaceae). Plant Systematic and Evolution 272: 211-222.

Las Peñas, M.L., G. Bernardello \& R. Kiesling. 2014. Classical and molecular cytogenetics and DNA content in Maihuenia and Pereskia (Cactaceae). Plant Systematic and Evolution 300: 549-558.

Las Peñas, M.L., R. Kiesling \& G. Bernardello. 2011. Karyotype, heterochromatin, and physical mapping of $5 \mathrm{~S}$ and 185.8-26S rDNA genes in Setiechinopsis (Cactaceae), an Argentine endemic genus. Haseltonia 9: 83-90.

Las Peñas, M.L., J.D. Urdampilleta, G. Bernardello \& E.R. Forni-Martins. 2009. Karyotypes, heterochromatin, and physical mapping of 18S-26S rDNA in Cactaceae. Cytogenetics Genome Research 124: 72-80.

Leitch, I.J. \& A.R. Leitch. 2013. Genome size diversity and evolution in land plants. In: I.J. Leitch, J. Greilhuber, J. Dolezel \& J. Wendel (eds.), Plant Genome Diversity, Vol. 2, pp. 307-320. Springer-Verlag, Vienna, Austria.

Levan, A, A. Sandberg \& K. Fredga. 1964. Nomenclature for centromeric position on chromosomes. Hereditas 52: 201220.

Majure, L., W. Judd, P. Soltis \& D. Soltis. 2012. Cytogeography of the Humifusa clade of Opuntia s.s. Mill. 1754 (Cactaceae, Opuntioideae, Opuntieae): correlations with Pleistocene refugia and morphological traits in a polyploid complex. Comparative Cytogenetic 6: 53-77.

Maluszynska, J. \& J. Heslop-Harrison. 1993. Molecular cytogenetics of the genus Arabidopsis: In situ localization of rDNA sites, chromosome numbers and diversity in centromeric heterochromatin. Annals of Botany 71: 479484.

Moreno, N., L. Stiefkens, M.L. Las Peñas, A. Bartoli, R. Tortosa \& G. Bernardello. 2012. Molecular cytogenetic studies in the "Xanthocephalum group" (Asteraceae). Plant Systematic and Evolution 298: 1503-1514.

Moscone, E.A., R. Samuel, T. Schwarzacher \& D. Schweizer. 2007. Complex rearrangements are involved in Cephalanthera (Orchidaceae) chromosome evolution. Chromosome Research 15: 931-943.

NAGL, W. 1978. Endopolyploidy and polyteny in differentiation and evolution: towards an understanding of quantitative and qualitative variation of nuclear DNA in ontogeny and phylogeny. North-Holland Publishing Company, Amsterdam. 283 pp.

Negron-Ortiz, V. 2007. Chromosome numbers, nuclear DNA content and polyploidy in Consolea (Cactaceae), an endemic cactus of the Caribbean Islands. American Journal of Botany 94: 1360-1370.

OHRI, D. 1998. Genome size variation and plant systematics.
Annals of Botany 82: 75-83.

Palomino, G. \& H.M. Heras. 2001. Karyotypic studies in Opuntia cochinera, O. hyptiacantha, and $O$. streptacantha (Cactaceae). Caryologia 54: 147-154.

Palomino, G., J. Dolezel, R. Cid, I. Brunner, I. Méndez \& A. Rubluo. 1999. Nuclear genome stability of Mammillaria san-angelensis (Cactaceae) regenerants induced by auxins in long term in vitro culture. Plant Science 141: 191-200.

Pereira de Castro, J. 2008. Números cromossômicos em espécies de Cataceae ocorrentes no nordeste do Brasil. Tesis. Mestrado em Agronomia. Universidade Federal da ParaíbaCentro de Ciências Agrárias. Paraíba, Brasil. 72 pp.

PIN, A.L. 1998. Nota sobre dos especies de Brasiliopuntia (Cactaceae): B. schulzii y B. brasiliensis. Rojasiana 4: 144-163.

PinkaVA, D.J. 2002. On the evolution of the North American Opuntioideae. In: D. Hunt \& N. Taylor (eds.), Studies in the Opuntioideae, pp. 59-98. Royal Botanic Gardens Kew, London, England.

Pinkava, D.J., M.A. Baker \& B.D. Parfitt. 1985. Chromosome number in some cacti of western North American-V. Systematic Botany 10: 471-483.

Pinkava, D.J., J.P. Rebman \& M.A. Baker. 1998. Chromosome numbers in some cacti of western North America-VII. Haseltonia 6: 32-41.

Powell, A.M. \& J.F. WeEdin. 2001. Chromosome numbers in Chihuahuan Desert Cactaceae. III. Trans-Pecos Texas. American Journal of Botany 88: 481-485.

Romero ZArco, C. 1986. A new method for estimating karyotype asymmetry. Taxon 35: 556-530.

Schwarzacher, T. \& P. Heslop-Harrison. 2000. Practical in situ hybridization. Bios Scientific Publishers Limited, Oxford. 203 pp.

Schweizer, D. \& P. Ambros. 1994. Chromosome banding. In: J.R. Gosden (ed.), Methods in Molecular Biology. Chromosome analysis protocols, pp. 97-112. Humana Press, Totowa, United States of America.

Segura, S., L. Scheinvar, G. Olalde, O. Leblanc, S. Filardo, A. Muratalla, C. Gallegos \& S. Flores. 2007. Genome sizes and ploidy levels in Mexican cactus pear species Opuntia (Tourn.) Mill. series Streptacanthae Britton et Rose, Leucotrichae DC., Heliabravoanae Scheinvar and Robustae Britton et Rose. Genetic Resources and Crop Evolution 54: 1033-1041.

Souza, M. \& A.N. Benko-Iseppon. 2004. Cytogenetics and chromosome banding patterns in Caesalpinioideae and Papilionioideae species of Pará, Amazonas, Brazil. Botanical Journal of the Linnean Society. 144: 181-191.

Wallace, R.S. \& S.L. Dickie. 2002. Systematic implications of chloroplast DNA sequences variation in the Opuntioideae. In: D.R. Hunt \& N.P. Taylor (eds.), Studies in the Opuntioideae (Cactaceae), pp. 9-24. David Hunt, Milborne Port, UK.

Zonneveld, B.J.M., I.J. Leitch \& M.D. Bennett. 2005. First nuclear DNA amounts in more than 300 angiosperms. Annals of Botany 96: 229-244. 